\title{
Clinical features, laboratory data, management and the risk factors that affect the mortality in patients with postoperative meningitis
}

\author{
Ilknur Erdem¹, Tayfun Hakan², Nurgul Ceran¹, Fatma Metin¹, Seniha Senbayrak Akcay³, \\ Metin Kucukercan ${ }^{3}$, M. Zafer Berkman'2, Pasa Goktas ${ }^{1}$ \\ ${ }^{1}$ Department of Infectious Disease and Clinical Microbiology, ${ }^{2}$ Neurosurgery, Microbiology and ${ }^{3}$ Clinical Microbiology, Haydarpasa Numune \\ Training and Research Hospital, Istanbul, Turkey
}

\begin{abstract}
Background: Nosocomial meningitis is a rare complication following neurosurgical procedures and is associated with high morbidity and mortality. Aim: The aim of this study was to describe the clinical characteristics and the risk factors associated with mortality in patients who developed nosocomial meningitis following neurosurgical operations. Setting and design: Tertiary care hospital and an observational study. Materials and Methods: The study subjects included 2265 patients who underwent various neurosurgical operations during 2003-05. The diagnosis of nosocomial meningitis was based on the Center for Disease Control criteria. Statistical analysis: It was performed by using Statistical Package for Social Sciences for Windows 10.0 program. Results: The incidence of postoperative nosocomial meningitis was $2.7 \%$ (62 episodes in 49 patients among 2265 patients operated). Staphylococcus aureus and Acinetobacter spp. were the most frequently isolated pathogens. Of the 49 with meningitis $20(40.8 \%)$ patients died. In the logistic regression analysis model, Glascow coma scale score less than 10 (Odds Ratio (OR): 19.419, 95\% Confidence Interval (CI); 1.637-230.41, $P=0.001$ ), and low cerebrospinal fluid glucose level ( $\leq 30 \mathrm{mg} / \mathrm{dL}$ ) (OR: 10.272, $95 \% \mathrm{Cl} ; 1.273-82.854, P=0.002)$, and presence of concurrent nosocomial infection (OR: 28.744, 95\% Cl;1.647-501.73, $P=0.001)$ were the independent risk factors associated with mortality. Conclusion: The mortality in patients who developed meningitis was high. The high percentage of concurrent nosocomial infections was associated with a high mortality rate which was a serious problem.
\end{abstract}

Key words: Meningitis, mortality, neurosurgery, nosocomial, postoperative

\section{Introduction}

Nosocomial meningitis is an uncommon hospitalacquired infection and is more frequently seen in neurosurgical clinics than in other clinics and is a life-threatening complication. Compared to communityacquired meningitis, nosocomial meningitis often has a more insidious onset, prolonged clinical course and is likely to be caused by resistant microorganisms. ${ }^{[1,2]}$ It is prudent to do lumbar puncture when the diagnosis of meningitis is suspected and the empiric antibiotic regime should be started according to the local guidelines, microbiological surveillance of local pathogens and resistance patterns of isolated microorganisms..$^{[2-4]}$ In this study, we report the clinical features, laboratory data, management and the risk factors associated wtih mortality in patients with postoperative meningitis who had neurosurgical operations in our neurosurgery clinic.

\section{Materials and Methods}

This is a prospective observational study and included 49 patients who developed postoperative meningitis among the 2265 patients who underwent various neurosurgical procedures between January 2003 and December 2005. Ours is a 750-bed hospital with a 39bed neurosurgery clinic. All operated patients received prophylactic antibiotics, cefazolin or cefriaxone, at the induction of anesthesia.

Lumbar puncture (LP) was performed in all the patients with suspected postoperative meningitis. Cerebrospinal fluid (CSF)and blood cultures were taken at the same time. Infection of other sites was 
excluded by taking various cultures. The specimens were cultured on 5\% sheep blood agar, EMB agar, chocolate agar. The anaerobic cultures were also done at $37^{\circ} \mathrm{C}$ for $24-48 \mathrm{~h}$ if needed. Microbiologic analysis of materials was performed using standard laboratory methods. Antimicrobial resistance patterns of isolated microorganisms was determined by the Kirby-Bauer disk diffusion method. ${ }^{[5]}$

The initial empiric antibiotic regimen was based on the most likely etiologic agent, if necessary, treatment was modified depending on the in vitro susceptibility pattern of the isolate. The data collected included age, gender, date of hospitalization, whether the operation was elective or an emergency, clinical characterestics, medical and/or surgical problems, laboratory results, isolated pathogens and the antimicrobial susceptibility patterns, treatment results, and the outcomes.

\section{Definitions}

The diagnosis of nosocomial meningitis was based on the Center for Disease Control (CDC) criteria. ${ }^{[6,7]}$ The inclusion criteria were as follows: 1) microorganism must have been isolated either from CSF culture or from blood cultures taken during the initial diagnostic workup; 2) clinical presentation with acute onset of fever, headache, stiff neck, meningeal signs, cranial nerve signs, or irritability; 3) elevated white blood cell count and elevated CSF protein and/or decreased glucose; and 4) organisms seen on Gram stain of CSF. Patients who did not meet the above criteria were excluded from the study.

Each episode of meningitis was considered as one case. Recurrent meningitis was considered when the patient had more than one episode of meningitis. Cure was defined as disappearance of all signs and symptoms of meningitis and sterilization of CSF cultures within 30 days. Failure of treatment was defined as meningitisrelated death or a relapse. Contamination was defined as growth of any microorganism from a CSF specimen in a patient without clinical features of meningitis.

\section{Statistical analysis}

Statistical analysis of data was performed using the Statistical Package for Social Sciences (SPSS) for Windows 10.0 program. Results were analyzed using $\chi^{2}$ test and Fisher's exact test. The risk factors that affect the mortality, which were found significant according to the univariate analysis, were evaluated by the multivariate analysis model. Odds ratio (OR) with $95 \%$ confidence interval (CI) was calculated. A $P$ value of less than 0.05 was considered to be statistically significant. A $P$ value of less than 0.001 was considered to be highly statistically significant.

\section{Results}

During the study period, we diagnosed 62 (2.7\%) postoperative meningitis episodes in 49 (2.1\%) patients among 2265 operated patients. The incidence of postoperative meningitis was $2.7 \%$. Twenty-six (53\%) of patients were male and the mean age $( \pm$ SD) was $43.06 \pm 25.6$ years. The indications for surgery were intracranial hemorrhage (20 patients), intracranial malignant tumors (14 patients), hydrocephalus (13 patients) and traumatic brain injury (two patients). The operations were performed as an emergency procedure in $28(57.1 \%)$ patients and as an elective procedure in 21 (42.9\%), Thirty-one (63\%) patients had external ventricular drainage (EVD). The mean period for development of postoperative meningitis was 7.8 days (range 3-21 days). Mean hospital stay was 46.5 days (range 7-289 days). Mean hospital stay in the neurosurgery clinic was 12.6 days.

While $40(82 \%)$ patients had a single episode, nine (18\%) patients had more than one episode and they were included in the "recurrent meningitis" category. Fever was observed in 45 (92\%) patients. The Glasgow Coma Scale (GCS) score was <10 in $14(28.6 \%)$ patients. Twenty-five (51\%) patients were stuporous and 14 (28.6\%) patients were unconscious. Ten $(20.4 \%)$ patients had bacteremia.

Fifty-three (85.4\%) cases had leukocytosis with shift to the left, $51(82.2 \%)$ cases had elevated erythrocyte sedimentation rate in the first hour, and all had increased C-reactive protein (CRP).

CSF cell count was 1000 cells per $\mathrm{mm}^{3}$ in 35 (56.5\%) examinations. CSF protein level was $\geq 200 \mathrm{mg} / \mathrm{dl}$ in 34 (54.8\%) cases, and CSF glucose level was $\leq 30 \mathrm{mg} / \mathrm{dl}$ in 25 (40.3\%) examinations. CSF cultures were positive in 53 (85\%) examinations [Table 1]. The isolated pathogens from CSF were Staphylococcus aureus (35.7\%), Acinetobacter spp. (mostly Acinetobacter baumanii) (30.3\%), Pseudomonas aeruginosa (10.7\%), coagulase negative staphylococci (CoNS) (8.9\%), Enterobacter spp. (5.4\%), Escherichia coli (3.6\%) and Candida spp. (5.4\%) [Table 2]. Fifteen (75\%) of $20 \mathrm{~S}$. aureus isolates and all of CoNS isolates were methicillin-resistant (MRSA).

All patients received appropriate antibiotic therapy. For patients with Acinetobacter meningitis, meropenem (3x2 g IV) + amikacin (1x1000 mg IV) were used in seven, cefoperazone-sulbactam (3x2 g IV) + amikacin (1x1000 mg IV) in seven and ciprofloxacin (3x400 mg IV) + amikacin (1x1000 mg IV) in two. One patient who had received empirical cefepime (3x2 g IV) + vancomycin (2x1g IV) died before specific antibiotherapy was started. Nine (52.9\%) of these patients with Acinetobacter meningitis died. Four of them were given meropenem treatment. Meropenem was also used in two patients with $E$. coli meningitis and both of them died. One patient with Enterobacter meningitis who had received 


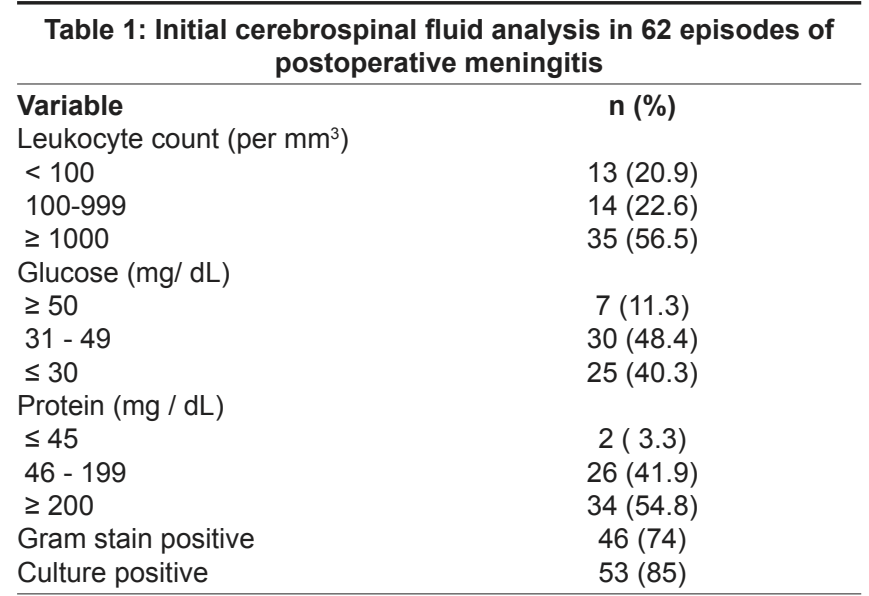

empirical cefepime (3x2 g IV) + vancomycin $(2 \times 1 \mathrm{~g}$ IV) treatment died before specific antibiotherapy was started. For the other two patients with Enterobacter meningitis, meropenem was used and one of them died. For $P$. aeruginosa meningitis, piperasillin-tazobactam ( $4 \times 4.5 \mathrm{~g} \mathrm{IV})$ was used in three patients, meropenem in two and ceftazidime ( $3 \times 2 \mathrm{~g} \mathrm{IV})$ in one. Of them, one patient who was on meropenem died. Vancomycin $(2 \times 1$ g IV and $10 \mathrm{mg}$ intrathecal) was given for patients with $S$. aureus and CoNS meningitis patients. Five (33\%) of the 15 patients with methicillin-resistant $S$. aureus (MRSA) meningitis died. Liposomal amphotericin B (3 mg/kg IV) was used for Candida meningitis. In consequence of late institution of antifungal treatment two patients with Candida meningitis died.

Of the 49 patients with postoperative nosocomial meningitis, 21 (42.8\%) had at least one concurrent nosocomial infection. The distribution of these infections was pneumonia in 11 (29.7\%), bacteremia in $10(27.1 \%)$, surgical site infection in six (16.2\%), urinary tract infection in six (16.2\%), and central venous catheter-related infection in four (10.8\%).

Of the 49 patients with postoperative meningitis, 20

\begin{tabular}{|c|c|}
\hline Microorganism & n (\%) \\
\hline S. aureus* & $20(35.7)$ \\
\hline Acinetobacter spp. & $17(30.3)$ \\
\hline aeruginosa ${ }^{* *}$ & $6(10.7)$ \\
\hline CoNS $S^{* * *}$ & $5(8.9)$ \\
\hline Enterobacter spp. & $3(5.4)$ \\
\hline E. colil ${ }^{* * * *}$ & $2(3.6)$ \\
\hline Candida spp. & $3(5.4)$ \\
\hline
\end{tabular}

*Staphylococcus aureus; ${ }^{* *}$ Pseudomonas aeruginosa; ${ }^{* * *}$ Coagulase negative staphylococci; ****Escherichia coli

(40.8\%) died. Of the 20 patients who died, 11 (55\%) had GCS score <10, 13 (65\%) had emergency surgery, $13(65 \%)$ had gram-negative infection $(P<0.05), 15$ (75\%) had CSF glucose level $<30 \mathrm{mg} / \mathrm{dL}$ ( $\mathrm{p}<0.05$ ), 17 $(85 \%)$ had CSF protein $>200 \mathrm{mg} / \mathrm{dL}(\mathrm{p}<0.05)$ and 15 $(75 \%)$ had at least one concurrent nosocomial infection $(P<0.05)$. On logistic regression analysis the mortality was significantly associated with the presence of a concurrent nosocomial infection (OR: 28.744, 95 \% CI; 1.647-501.73, $P=0.001$ ), GCS score $<10$ (OR: 19.419, $95 \% \mathrm{CI} ; 1.637-230.41, P=0.001$ ), and the CSF glucose level $\leq 30 \mathrm{mg} / \mathrm{dL}$ (OR: 10.272, 95\% CI 1.273-82.854, $P=0.002$ ). In patients having postoperative meningitis, the presence of concurrent nosocomial infection increased the mortality rate by 28 times. The GCS score $<10$ was associated with increased mortality rate by 19.4 times. CSF glucose $<30 \mathrm{mg} / \mathrm{dL}$ was associated with increased mortality rate by 6.6 times [Tables 3 and 4].

\section{Discussion}

Meningitis and shunt infections are the most common nosocomial central nervous system infections. Most of all nosocomial meningitis develop postoperatively. ${ }^{[1-3,8]}$ In our study the incidence of postoperative meningitis was $2.7 \%$. The reported incidence of postoperative meningitis was $1-3.5 \% \cdot{ }^{[9-13]}$ This variable incidence

\begin{tabular}{|c|c|c|c|c|c|c|c|}
\hline \multirow{3}{*}{ Variable } & \multirow{2}{*}{\multicolumn{2}{|c|}{ Died }} & \multirow{2}{*}{\multicolumn{2}{|c|}{ Survived }} & \multirow{2}{*}{\multicolumn{2}{|c|}{ Total }} & \multirow{3}{*}{$P$ value } \\
\hline & & & & & & & \\
\hline & $\mathbf{n}$ & $\%$ & n & $\%$ & $\mathbf{n}$ & $\%$ & \\
\hline \multicolumn{8}{|l|}{ Gender } \\
\hline Female & 9 & 45.0 & 14 & 48.3 & 23 & 46.9 & \multirow[t]{2}{*}{0.821} \\
\hline Male & 11 & 55.0 & 15 & 51.7 & 26 & 52.1 & \\
\hline Old age (above 60 years) & 9 & 45.0 & 7 & 24.1 & 16 & 32.7 & 0.126 \\
\hline Emergency operation & 13 & 65.0 & 15 & 51.7 & 28 & 57.1 & 0.356 \\
\hline GCS $\leq 10$ & 11 & 55.0 & 3 & 10.3 & 14 & 28.6 & 0.001 \\
\hline The presence of intracranial haemorragia & 11 & 55.0 & 9 & 31.0 & 20 & 40.8 & 0.093 \\
\hline Concurrent nosocomial infection & 15 & 75.0 & 6 & 20.7 & 21 & 42.9 & 0.001 \\
\hline Concurrent nosocomial bacteremia & 7 & 35.0 & 3 & 10.3 & 10 & 20.4 & 0.035 \\
\hline Concurrent nosocomial pneumonia & 8 & 40.0 & 3 & 10.3 & 11 & 22.4 & 0.014 \\
\hline Gram-negative etiology & 13 & 65.0 & 13 & 44.8 & 26 & 53.1 & 0.164 \\
\hline Isolation of Acinetobacter spp. & 9 & 45.0 & 8 & 27.6 & 17 & 34.7 & 0.208 \\
\hline Isolation of MRSA* & 5 & 25.0 & 10 & 34.5 & 15 & 30.6 & 0.479 \\
\hline CSF protein level $\geq 200 \mathrm{mg} / \mathrm{dl}$ & 17 & 85.0 & 15 & 51.7 & 32 & 65.3 & 0.016 \\
\hline CSF glucose level $\leq 30 \mathrm{mg} / \mathrm{dl}$ & 15 & 75.0 & 9 & 31.0 & 24 & 49.0 & 0.002 \\
\hline
\end{tabular}

*Methicillin-resistant Staphylococcus aureus 


\begin{tabular}{lcc}
\hline $\begin{array}{l}\text { Table 4: The multivariant analysis results of significant risk fac- } \\
\text { tors for mortality }\end{array}$ \\
\hline Factors & Odds ratio & $\begin{array}{c}\mathbf{9 5 \%} \text { confidence } \\
\text { interval }\end{array}$ \\
GCS $\leq 10$ & 32.400 & $3.277-320.360$ \\
Concurrent nosocomial infection & 19.556 & $3.315-115.372$ \\
CSF glucose level $\leq 30 \mathrm{mg} / \mathrm{dl}$ & $6, .00$ & $1.229-35.438$ \\
\hline
\end{tabular}

rates are partly related to methodological issues like definitions and surveillance methods. Some studies included all craniotomies (i.e. clean, contaminated, and dirty surgical sites), whereas others select only clean craniotomies. ${ }^{[2,14,15]}$ The reported risk factors for nosocomial meningitis include postoperative CSF leakage, intraventricular shunt operations, external ventricular drainage, repeated operation, amd emergency surgery.$^{[9,10,16-18]}$ This study had not assessed the risk factors for postoperative nosocomial meningitis, however, nosocomial meningitis was more frequently seen in patients who had emergent surgery or EVD.

$S$. aureus and CoNS are known to be frequent pathogens in postoperative nosocomial meningitis, particulary involving intracranial devices. Gramnegative bacterial meningitis is usually related to neurosurgical procedures or head trauma. It is usually hospital-acquired and the reported incidence has increased significantly in the recent years. ${ }^{[17-26]}$ Both the widespread use of broad-spectrum antibiotics and the deterioration of the clinical status have been shown to favor colonization of hospitalized patients by gram-negative bacteria. In recent years nonfermentative gram-negative bacteria, most commonly Acinetobacter spp. and $P$. aeruginosa, have gained importance as hospital pathogens..$^{[9,26-29]}$ In the present study, the most commonly isolated agents were Acinetobacter spp. and MRSA. These strains are endemic in our hospital.

Treatment of postoperative meningitis is based on the same general principles guiding management of community-acquired meningitis. Initial empiric antibiotic therapy should achieve adequate therapeutic levels in CSF and cover aerobic gram-negative bacilli including $P$. aeruginosa and $S$. aureus. When CSF culture reveals a particular bacterial pathogen, treatment should be directed towards the specific pathogen depending on the sensitivity. ${ }^{[2,4,8,23]}$ In our study, treatment was based on antimicrobial susceptibility results of isolated pathogens. Two patients with Acinetobacter meningitis received ciprofloxacin because these strains were only susceptible to ciprofloxacin.

Nosocomial meningitis is a severe infection with significant morbidity and mortality. It is difficult to establish how the infection affects the mortality in neurosurgery clinics in the presence of concurrent medical problems. ${ }^{[3]}$ The type of microorganism that caused meningitis, primary brain disease, initial consciousness level, very low CSF glucose concentration, presence of bacteremia, inappropriate antibiotic use are reported as important risk factors of mortality ${ }^{[9,10,20,29,30]}$ In our study, the mortality was high. On logistic regression analysis the independent variables of mortality were level of consciousness (GCS $<10$ ), presence of concurrent nosocomial infection, and CSF glucose level $<30 \mathrm{mg} / \mathrm{dL}$. Our results suggest that one should look for the presence of other concurrent nosocomial infections and it should be effectively treated as it is likely to be associated with high mortality. An effective infection control program is necessary to prevent nosocomial infections.

In conclusion, postoperative nosocomial meningitis should be suspected in patients with a new onset fever or an unexplained change in neurologic status. Early diagnosis and choice of appropriate antibiotics according to epidemiologic trends are the key issues to reduce mortality and morbidity. A good surveillance should be in place in postoperative care patients to diagnose, treat and prevent these infections.

\section{References}

1. Reingold AL, Broome CV. Nosocomial central nervous sytem infections. In: Bennett JV, Brachman PS, editors. Hospital infections. 3rd ed. Boston: Little, Brown and Company; 1992. p. 673-85.

2. Morris A, Low DE. Nosocomial bacterial meningitis, including central nervous system shunt infections. Infect Dis Clin North Am 1999;13:735-50.

3. Kim JM, Park ES, Jeong JS, Kim KM, Kim JM, Oh HS, et al. Multicenter surveillance study for nosocomial infections in major hospitals in Korea. Am J Infect Control 2000;28:454-8.

4. Meena AK, Prasad VS, Murthy JM. Neurological intensive care in India: Disease spectrum and outcome. Neurol India 2001;49:S1-7.

5. Murray PR, Baron EJ, Pfaller MA, Tenover FC, Yolken RH. Manual of Clinical Microbiology. 7th ed. Washington DC: ASM press; 1999.

6. Horan TC, Gaynes RP, Martone WJ, Jarvis WR, Emori TG. CDC definitions of nosocomial surgical site infections, 1992: A modification of CDC definitions of surgical wound infections. Am J Infect Control 1992;20:271-4.

7. Horan TC, Gaynes RP. Surveillance of nosocomial infections. In: Mayhall CG, editor. Hospital Epidemiology and Infection Control. 3rd ed. Philadelphia: Lippincott Williams and Wilkins; 2004. p. 1659-702.v

8. Blomstedt GC. Infections in neurosurgery: A retrospective study of 1143 patients and 1517 operations. Acta Neurochir (Wien) 1985;78:81-90.

9. Kremery V, Paradisi F, Pediatric Nosocomial Meningitis Study Group. Nosocomial bacterial and fungal meningitis in children: An eight year national survey reporting 101 cases. Int J Antimicrob Agents 2000;15:143-7.

10. Federico G, Tumbarello M, Spanu T, Rosell R, Iacoangeli M, Scerrati M, et al. Risk factors and prognostic indicators of bacterial meningitis in a cohort of 3580 postneurosurgical patients. Scand J Infect Dis 2001;33:533-7.

11. Hussein AS, Shafran SD. Acute bacterial meningitis in adults. Medicine 2000;79:360-8.

12. Palabiyikoglu I, Tekeli E, Cokca F, Akan O, Unal N, Erberktas I, et al. Nosocomial meningitis in a university hospital between 1993 and 2002. J Hosp Infect 2006;62:94-7.

13. McClelland S 3rd, Hall WA. Postoperative central nervous system infection: Incidence and associated factors in 2111 neurosurgical procedures. Clin Infect Dis 2007;45:55-9.

14. Kourbeti IS, Jacobs AV, Koslow M, Karabetsos D, Holzman RS. Risk factors associated with postcraniotomy meningitis. Neurosurgery 2007;60:317-25. 
15. Reichert MC, Medeiros EA, Ferraz FA. Hospital- acquired meningitis in patients undergoing craniotomy: Incidence, evolution, and risk factors. Am J Infect Control 2002;30:158-64.

16. Korinek AM. Risk factors for neurosurgical site infections after craniotomy: A prospective multicenter study of 2944 patients, The French Study Group of Neurosurgical Infections, Risk factors for neurosurgical site infections after craniotomy: A prospective multicenter study of 2944 patients. Neurosurgery 1997;41:1073-81.

17. Streharova A, Benca J, Holeckova K, Balik J, Sula I, Lesnakova A, et al. Comparison of postsurgical and community acquired bacterial meningitis: Analysis of 372 cases within a nationwide survey. Neuro Endocrinol Lett 2007;28:7-9.

18. Weisfelt M, van de Beek D, Spanjaard L, de Gans J. Nosocomial bacterial meningitis in adults: A prospective series of 50 cases. J Hosp Infect 2007;66:71-8.

19. Ross D, Rosegay H, Pons V. Differentiation of aseptic and bacterial meningitis in postoperative neurosurgical patients. J Neurosurg 1988;69:669-74.

20. Wang KW, Chang WN, Huang CR, Tsai NW, Tsui HW, Wang HC, et al. Post-neurosurgical nosocomial bacterial meningitis in adults: Microbiology, clinical features, and outcomes. J Clin Neurosci 2005; $12: 647-50$.

21. Benca J, Ondrusova A, Kisac P, Krcmery V. Postoperative meningitis: Shift in etiology? Neuro Endocrinol Lett 2007;28:22.

22. Pintado V, Meseguer MA, Fortun J, Cobo J, Navas E, Quereda C, et al. Clinical study of 44 cases of Staphylococcus aureus meningitis. Eur J Clin Microbiol Infect Dis 2002;21:864-8.

23. Hodges GR, Perkins RL. Hospital-associated bacterial meningitis. Am
J Med Sci 1976;271:335-41.

24. Tunkel AR, Hartman BJ, Kaplan SL, Kaufman BA, Roos KL, Scheld WM, et al. Practice guidelines for the management of bacterial meningitis. Clin Infect Dis 2004;39:1267-84.

25. Durand ML, Calderwood SB, Weber DJ, Miller SI, Southwick FS, Caviness VS Jr, et al. Acute bacterial meningitis in adults: A review of 493 episodes. N Engl J Med 1993;328:21-8.

26. O'Neill E, Humphreys H, Phillips J, Smyth EG. Third-generation cephalosporin resistance among Gram-negative bacilli causing meningitis in neurosurgical patients: Significant challenges in ensuring effective antibiotic therapy. J Antimicrob Chemother 2006;57:356-9.

27. Gulati S, Kapil A, Das B, Dwivedi SN, Mahapatra AK. Nosocomial infections due to Acinetobacter baumannii in a neurosurgery ICU. Neurol India 2001;49:134-7.

28. Huang CR, Lu CH, Chuang YC, Tsai NW, Chang CC, Chen SF, et al. Adult Pseudomonas aeruginosa meningitis: High incidence of underlying medical and/or postneurosurgical conditions and high mortality rate. Jpn J Infect Dis 2007;60:397-9.

29. Mombelli G, Klastersky J, Coppens L, Daneau D, Nubourgh Y. Gram negative bacillary meningitis in neurosurgical patients. J Neurosurg 1983;58:634-41.

30. Lu CH, Chang WN, Chuang YC, Chang HW. Gram-negative bacillary meningitis in adult post-neurosurgical patients. Surg Neurol $1999 ; 52: 438-43$.

Accepted on 30-10-2008

Source of Support: Nil, Conflict of Interest: None declared. 